\title{
DISTANCIA RECORRIDA MEDIANTE LA PRUEBA DE CAMINATA DE 6 MINUTOS EN ADULTOS MAYORES SALUDABLES ENTRE 60 Y 80 AÑOS
}

\author{
DISTANCE COVERED BY WALK TEST 6 MINUTES BETWEEN \\ HEALTHY SENIORS 60 TO 80 YEARS OLD
}

\author{
SANTOS CHERO PISFIL \\ Universidad Norbert Wiener \\ RENZO EDUARDO DÍAZ RAMÍREZ \\ Universidad Norbert Wiener \\ JESÚS JONATHAN QUISPE RAMÍREZ \\ Universidad Norbert Wiener
}

\section{RESUMEN}

El gran desafío de la salud pública en el grupo etario de 60 a 80 años es promover el mantenimiento de la funcionalidad. La OMS considera que entre 2000 y 2050 la proporción de los habitantes del planeta mayores de 60 años se duplicará, pasando del $11 \%$ al $22 \%$. El Perú tiene más de 2,9 millones de habitantes mayores de 60 años, según cifras del Ministerio de Salud. Esta población ha aumentado en $39 \%$ en los últimos diez años, y seguirá creciendo. Muchos realizan poca actividad física, es decir, son adultos sedentarios; por ello, importa conocer cuál es el comportamiento de los mayores de 60 ańos a través de la prueba de caminata de 6 minutos, información que constituye un instrumento que estima la capacidad funcional, y evalúa capacidad y tolerancia. Este procedimiento se ajusta a las necesidades de nuestro grupo de estudio, pues se trata de una prueba relacionada con el quehacer diario, de tipo submáximo, y que es posible que la realicen nuestros pacientes, al tiempo que nos ayuda a diseñar programas de entrenamiento de acuerdo a su condición.

El objetivo fue determinar la distancia recorrida mediante la prueba de caminata de 6 minutos en adultos mayores saludables de 60 a 80 ańos, divididos por grupos etarios, en grupo I (60-70) y grupo II (71-80).

Estudio descriptivo, de diseńo observacional, en pacientes que acuden al programa de prevención en el adulto mayor. Muestra compuesta por 43 adultos mayores saludables de entre 60 y 80 ańos: 21 varones y 22 mujeres. Se realizaron dos pruebas. Se utilizó el programa estadístico SPSS versión 18.

En distancia media: $414,6 \pm 88,8$ metros, los hombres registraron una distancia recorrida promedio de 432,2 \pm 75,7 metros, mayor que las mujeres, quienes registraron una distancia recorrida de 399,4 \pm 97,9 metros.

Los resultados obtenidos en la distancia recorrida mediante la PC6M muestran que los varones recorren mayor distancia que las mujeres, y la distancia disminuye conforme la edad aumenta. Se pueden diseñar programas de entrenamiento mediante caminatas respecto a tiempo y distancia.

Palabras clave: PC6M (prueba de caminata de 6 minutos), FC (frecuencia cardiaca), PA (presión arterial), IMC (índice de masa corporal), escala de Borg. 


\section{ABSTRACT}

The great challenge of public health in this age group is to promote the maintenance of functionality. The WHO estimates that between 2000 and 2050 the proportion of the world population over 60 years old will double, from $11 \%$ to $22 \%$. Peru has more than 2.9 million people over 60 years old, according to the Ministry of Health. This population has increased by $39 \%$ in the last ten years, it will continue to grow, many of them do little physical activity which leads to have sedentary adults, for that reason is important to know what is the behavior of those over 60 years old through the test walk in 6 minutes. This is a tool that estimates the functional capacity and assesses the capacity and tolerance, therefore it adjusted to the needs of our study group to be a test related to the daily work of submaximal type and it is possible to realize in our patients, also helps us to design training programs according to their condition. The objective was to determine the distance traveled by testing 6-minute walk in healthy adults over 60 to 80 years old, divided by age groups, group I (60 to 70 years old) and group II (71 to 80 years old). Materials and methods: descriptive, observational design. Patients attending prevention program in the elderly. Sample of 43 healthy adults aged 60-80 years old, 21 men and 22 women, two tests were performed. SPSS version 18 was used. Result: average distance $414.6 \pm 88.8$ meters; It is the male gender who obtained an average distance of $432.2 \pm 75.7$ meters; greater than those of the female gender who obtained a distance of $399.4 \pm 97.9$ meters. Conclusions: The results of the distance traveled by 6MWD show that males roam farther than women, the gap decreases as age increases. You can design training programs through hikes based on time and distance.

Key words: 6MWD test (6 minute walk), FC (heart rate), BP (blood pressure), BMI (body mass index), Borg scale.

\section{INTRODUCCIÓN}

En 2012, la Organización Mundial de la Salud (OMS) dedica el Día Mundial de la Salud al envejecimiento saludable y la salud de las personas saludables con el lema "La salud añade vida a los años". La celebración del Día Mundial de la Salud tiene como fin concientizar no solo en los desafíos que acompañan al envejecimiento de la población, sino también acerca de las oportunidades que se pueden desarrollar para ella ${ }^{1}$.

En atención primaria, los médicos necesitan pruebas de bajo costo que permitan medir la capacidad funcional y limitación en personas de edad avanzada. La prueba de caminata de 6 minutos fue desarrollada para evaluar la capacidad funcional, monitorear la efectividad de tratamientos diversos y establecer el pronóstico de pacientes con enfermedades cardiorrespiratorias; y pacientes con tales disfunciones que presentan intolerancia al ejercicio debido al mal funcionamiento de los sistemas respiratorio o cardiovascular, así como disfunción de los músculos esqueléticos periféricos y respiratorios ${ }^{2}$. Por tanto, la mayoría de personas de edad avanzada puede realizar con seguridad la prueba de caminata de 6 minutos en el ámbito ambulatorio. Actualmente, en el Perú existen valores referenciales solamente del grupo comprendido entre los 20 y 60 ańos, en personas adultas saludables ${ }^{3}$.

El objetivo de este estudio es obtener la distancia recorrida con la prueba de caminata de 6 minutos en la población adulta mayor saludable en nuestro país de 60 a 80 años, con la finalidad de establecer parámetros en esta población, así como para obtener diagnósticos funcionales, conocer la evolución y desarrollar programas de tra- 
tamiento de actividad física; ello, respecto a su funcionabilidad en personas saludables asi como también en personas con patologías cardiorrespiratorias, musculoesqueléticas, etcétera.

\section{MATERIALES Y MÉTODOS}

Muestra conformada por 43 adultos mayores saludables entre 60 y 80 años, 20 varones y 23 mujeres. Los criterios de inclusión fueron: adulto mayor que tenga la edad correspondiente, presión arterial controlada, deambulación sin ninguna restricción, capacidad para realizar sus actividades habituales. Los criterios de exclusión: adulto mayor con patología cardiaca demostrada, con ayuda biomecánica, con hábitos de beber alcohol y fumar, con enfermedades neurológicas degenerativas o enfermedades respiratorias crónicas.

\section{Preparación del paciente}

Se les brindó una charla previa en la que se les explicó la preparación: traer calzado apropiado, ropa ligera, y abstenerse de ejercicio vigoroso durante la semana.

\section{PROCEDIMIENTO}

La prueba se realizó en un patio, con espacio cerrado. La distancia que se utilizó fue de 30 metros, con marcadores cada 5 metros, y 2 conos de $50 \mathrm{~cm}$ antes del inicio y final de la distancia recomendada. Como medida de precaución se acondicionó un ambiente de primeros auxilios para una intervención rápida ${ }^{4}$.

Antes de la aplicación de la prueba de caminata de 6 minutos a los adultos mayores se realizó la recolección de datos; se les solicitó permanecer en reposo por $30 \mathrm{mi}$ nutos y posteriormente se aplicó la escala subjetiva de Borg modificada, pidiéndoles que calificaran la sensación subjetiva de falta de aire (disnea) y sensación de cansancio en miembros inferiores. Seguidamente, se registró la FC, PA, Sp02 a través de un tensiómetro y un pulso oxímetro 5 .

$\mathrm{Al}$ término de la recolección de signos se procedió a indicar las instrucciones previas y el recorrido por donde debería realizar la prueba. La ejecución de las pruebas se realizó según el protocolo descrito por la ATS; después de un descanso de $30 \mathrm{mi-}$ nutos se tomó la segunda prueba con las mismas pautas establecidas anteriormente.

\section{Plan de procesamiento de datos}

Los datos obtenidos fueron procesados electrónicamente por el programa SPSS versión 18.

\section{RESULTADOS}

La distancia recorrida promedio fue de 414,6 metros con desviación estándar \pm 88,8 metros, la distancia mínima fue de 210 metros y la distancia máxima recorrida, de 568 metros.

En relación al género, los varones alcanzaron una distancia media de 432,2 \pm 75,7 metros; con una distancia mínima de 245 metros y una máxima de 540 metros. Las mujeres recorrieron $399,4 \pm 97,9$ metros; la distancia mínima recorrida fue de 210 metros y la distancia máxima, de 565 metros. Los del grupo etario I recorrieron mayor distancia (455.40 vs 403.8), los que presentaron mayor peso fueron los del grupo II $(67.8 \pm 16.3$ vs $65.6 \pm 10.4)$; la talla fue un factor influyente: el grupo II registró medidas menores $(1.57 \pm 0.1$ vs $1.63 \pm 0.1)$, además, el IMC de mayor predominio estuvo en el grupo II $(27.4 \pm 6.6$ vs $24.8 \pm 3.9)$. 


\section{DISCUSIÓN}

La prueba de caminata de 6 minutos ha sido descrita como la submáxima ideal para evaluar la capacidad funcional de pacientes con patologías cardiorrespiratorias, debido a su utilidad, economía y facilidad, así como su relación con las actividades de la vida diaria. En este estudio se consideraron a adultos mayores de 60 a 80 años de edad de ambos géneros; se consideró peso, talla e IMC. Osees ${ }^{6}$ refiere que los resultados en la distancia recorrida fueron $644 \pm 84$ metros en varones y $576 \pm 87$ en mujeres, lo que en comparación con nuestro estudio (pacientes varones 432,2 $\pm 75,7$ metros y mujeres, 399, $4 \pm 97,9$ metros) revela una diferencia aproximadamente de $33 \%$ en varones y $31 \%$ en mujeres.

Sin embargo, Bautmans et al. ${ }^{7}$ refieren que su estudio reveló la distancia promedio fueron $603 \pm 80$ metros y la diferencia con los peruanos es 189 metros aproximadamente $32 \%$ de la distancia recorrida. Los resultados obtenidos por Bernadine et al. ${ }^{8}$ revelan $659 \pm 62$ metros de distancia recorrida; la diferencia con el presente estudio es de 245 metros, lo que representa un $38 \%$ aproximadamente. En ambos sexos se evidencia que la talla, el peso y el IMC son factores que han influenciado en la distancia recorrida por nuestros pacientes.

\section{CONCLUSIONES}

Los resultados obtenidos en la distancia recorrida mediante la PC6M muestran que los varones recorren mayor distancia que las mujeres.

La distancia va disminuyendo conforme la edad aumenta.

La talla y el IMC son factores influyentes sobre la distancia a recorrer.
Se pueden diseñar programas de entrenamiento mediante caminatas en base a tiempo y distancia.

La prueba sirve de medio de referencia al comparar caminatas de 6 minutos en pacientes afectados con enfermedades cardiorrespiratorias crónicas.

Es posible conocer el grado de funcionalidad en base a la distancia recorrida.

\section{REFERENCIAS BIBLIOGRÁFICAS}
1. Minsa [Internet]. Perú: Minsa 2012 [Actua- lizado 17-4-2013. Consulta: 8 de julio de 2014. Disponible en: <http://www. minsa.gob.pe>.

2. Enright PL et al. The 6-min walk test: a quick measure of functional status in elderly adults. Chest. February 2003; 123:387-98).

3. Gamarra Torrejón R, Mendoza Román M. Distancia recorrida mediante la prueba de caminata de 6 minutos en personas saludables de 20 a 60 años de un hospital de la ciudad de Lima en los meses de setiembre, octubre y noviembre 2011 Lima, Perú: Universidad Norbert Wiener; 2011.

4. Giraldo Estrada H. EPOC: diagnóstico y tratamiento integral. $3^{\mathrm{a}}$ ed. Bogotá: Editorial médica panamericana. 2008.

5. ATS, American Thoracic Society. ATS Statement: Guidelines for the Six-Minute Walk Test. This official statement of the american thoracic sciety was approved by the ATS, March 2002: 1-7.

6. Osses R et al. RevMed Chile 2010; 138: 1124-1130.

7. Bautmans et al. BMC geriatrics, BruselasBélgica, 2014.

8. Bernadine et al. Rev. Respiratory MedicineAustralia. 2005. 